\title{
American Contingent Claims under Small Proportional Transaction Costs
}

\author{
Krzysztof Tokarz ${ }^{1)}$ and Tomasz Zastawniak ${ }^{2)}$ \\ 1) Graduate School of Business, National-Louis University \\ ul. Zielona 27, 33-300 Nowy Sacz, Poland \\ ktokarz@wsb-nlu.edu.pl \\ 2) Department of Mathematics, University of York \\ Heslington, York YO10 5DD, United Kingdom \\ tz506@york.ac.uk
}

\begin{abstract}
American options are considered in the binary tree model under small proportional transaction costs. Dynamic programming type algorithms, which extend the Snell envelope construction, are developed for computing the ask and bid prices (also known as the upper and lower hedging prices) of such options together with the corresponding optimal hedging strategies for the writer and for the seller of the option. Representations of the ask and bid prices of American options in terms risk-neutral expectations of stopped option payoffs are also established in this setting.
\end{abstract}

\section{Introduction}

In the presence of small proportional transaction costs in the form of a bidask spread of the underlying stock prices we shall consider American options exercised by the physical delivery of a portfolio of cash and stock.

For example, when a call option with strike price $K$ is exercised by physical delivery, the holder of the option pays $K$ and receives 1 share, that is, the option is exercised by the delivery of a portfolio $(-K, 1)$ of cash and stock. If buying or selling stock incurs transaction costs, then physical delivery is not equivalent to cash settlement.

Chalasani and Jha CJ01 investigated American options with cash settlement only under (not necessarily small) proportional transaction costs. They obtained general representations involving so-called randomised stopping times for the ask and bid prices of American contingent claims. However, no algorithmic procedure for computing the prices was proposed. Indeed, Chalasani and Jha commented that "The computation of the expressions for the upper and 
lower hedging prices appears non-trivial. It would be useful to design efficient algorithms for approximating the values of these expressions." (CJ01, p. 72.)

Here we put forward two algorithms extending the Snell envelope construction, one for computing the ask price (the upper hedging price) and one for the bid price (the lower hedging price) of an arbitrary American contingent claim under small proportional transaction costs in the binary tree model. We also construct optimal hedging strategies for the option writer as well as for the seller, and establish representations of the ask and bid option prices in terms of risk-neutral expectations of stopped payoffs. In contrast to Chalasani and Jha CJ01, our results involve ordinary rather than randomised stopping times, and we consider American options with physical delivery rather than ones with cash settlement only.

Numerical examples demonstrating the applicability of the pricing algorithms are provided, and certain novel and interesting features are noted, such as the non-equality of bid prices for American and European calls, or different optimal stopping times for the writer and the holder of an American option under transaction costs.

Other papers devoted to American options under proportional transaction costs include Davis and Zariphopoulou DZ95, Levental and Skorohod [LS97, Mercurio and Vorst [MV97, Kociński [Koc99, Koc01, Perrakis and Lefoll PL00, PL04, Constantinides and Zariphopoulou CZ01, Jakubenas, Levental and Ryznar [JLR03, and Constantinides and Perrakis CP04.

For a wider context we refer to the extensive literature concerned with pricing and hedging European options under proportional transaction costs. This problem was studied by Merton [Mer90], Dermody and Rockafellar [DR91, Boyle and Vorst BV92, Bensaid, Lesne, Pagès and Scheinkman BLPS92, Edirsinghe, Naik and Uppal [ENU93, Jouini and Kallal JK95, Kusuoka Kus95, Naik [Nai95], Shirakawa and Konno [SK95, Soner, Shreve and Cvitanić [SSC95], Cvitanić and Karatzas [CK96, Koehl, Pham and Touzi [KPT96, [KPT99, [KPT01, Cvitanić, Pham and Touzi CPT99, Levental and Skorohod [LS97, Perrakis and Lefoll [PL97, Stettner Ste97, Ste00, Rutkowski Rut98, Touzi [Tou99, Jouini [Jou00, Ortu Ort01], Palmer Pal01a, Pal01b, Kociński Koc04, and many others.

In the majority of these papers the authors assume a stock price process $S_{t}$ under no-arbitrage conditions, and introduce transaction costs by multiplying $S_{t}$ by constant factors $1+\lambda$ and $1-\mu$ for some $\lambda, \mu \geq 0$. Here we follow the more general approach of Jouini and Kallal JK95 involving bid-ask spreads $S_{t}^{b} \leq S_{t}^{a}$ for the stock price. As pointed out by Jouini [Jou00], the spreads can be interpreted as proportional transaction costs in the above sense, but can also be explained by the buying and selling of limit orders. Accordingly, $S_{t}^{a}$ and $S_{t}^{b}$ can be thought of as the prices ensuring liquidity in the stock market, that is, at which stock can be bought or, respectively, sold on demand. The spreads, therefore, include proportional transaction costs, but are not limited to them.

The lack of arbitrage in a model with bid-ask spreads was characterised by Jouini and Kallal [JK95] in terms of the existence of suitably defined risk-neutral measures and associated stock price processes, see Theorem 2.1 below. We use 
their results here as our starting point. See also Ortu Ort01.

Small proportional transactions costs similar to those considered in the present paper have been studied by a number authors in various contexts, for example, by Bensaid, Lesne, Pagès and Scheinkman BLPS92, Koehl, Pham and Touzi KPT99, Kociński [Koc99, [Koc01, and Melnikov and Petrachenko MP05. The definitions of small transaction costs differ slightly between these various approaches, but there is a substantial overlap, also with that adopted in the present paper.

Even in the relatively simple case of small proportional transaction costs within the binary tree model the results for American options give rise to interesting and perhaps unexpected effects such as, for example, the non-equality of bid prices of American and European calls. The algorithms developed can inform further research aiming to extend the results to American options under general proportional transaction costs and to more general market models.

Small transaction costs can be considered as perturbations of the frictionfree model. From a practical point of view the assumption of small proportional transaction costs might become a limitation if the time step is chosen to be very short, since transaction costs would then need to scale down to nil asymptotically. The difficulties inherent in continuous time models under transaction costs, which are known to lead to unrealistic option prices and trivial hedging strategies (see Soner, Shreve and Cvitanić SSC95, and Cvitanić, Pham and Touzi [CPT99] for European options, and Levental and Skorohod [LS97] for American options), indicate that asymptotically short time steps may in fact be incompatible with proportional transaction costs.

Leland [Lel85] and Hoggard, Whalley and Wilmott [HWW94 have sought a compromise solution by combining the continuous time model under proportional transaction costs with hedging portfolios rebalanced only at discrete time instances, with a fixed finite time interval $h$ between them. Leland type approaches are limited by the condition $\sqrt{\frac{2}{\pi}} \frac{2 k}{\sigma \sqrt{h}}<1$, the expression on the left known as the Leland number, where $2 k$ is the round-trip transaction cost rate (expressed as a percentage of the stock price) and $\sigma$ is the volatility of the underlying asset, see Avellaneda and Parás AP94. (Observe that the round-trip transaction cost rate $k$ in AP94 is the same as $2 k$ in our notation.) None of these Leland type approaches have been extended to American options, inviting further research in this area of great practical importance. It will be demonstrated in Section 4 that our small transaction costs assumption asymptotically yields a very similar restriction, $\frac{2 k}{\sigma \sqrt{h}}<1$. The results of the present paper can thus be regarded as a discrete version of Leland type approaches as applied to American options.

The paper is organised as follows: In Section 2 we describe the model, introduce some notation, basic notions and facts, and specify the small proportional transaction costs assumption. The main results are presented in Section 3 , with proofs in the Appendix. Numerical examples demonstrating an application of the pricing algorithms are discussed in Section 4 . This section also touches upon the relationship between American and European call prices under transaction 
costs. Section 5 concludes.

\section{Model Specifications}

We adopt the binary tree model with trading times $t=0, \ldots, T$ for some fixed positive integer $T$. The corresponding probability space $\Omega$ consists of sequences $\omega^{1} \omega^{2} \cdots \omega^{T}$ with $\omega^{1}, \ldots, \omega^{T} \in\{\mathrm{u}, \mathrm{d}\}$, where $\mathrm{u}$ and $\mathrm{d}$ stand for $u p$ and down. We take $\mathcal{F}$ to be the $\sigma$-field consisting of all subsets of $\Omega$, and $\mathbb{Q}$ to be a probability measure on $\mathcal{F}$ such that $\mathbb{Q}\{\omega\}>0$ for each $\omega \in \Omega$.

For each $t=1, \ldots, T$ we define a random variable $\eta_{t}: \Omega \ni \omega^{1} \omega^{2} \cdots \omega^{T} \mapsto$ $\omega^{t} \in\{\mathrm{u}, \mathrm{d}\}$. A node $\omega_{t}=\omega^{1} \omega^{2} \cdots \omega^{t}$ of the tree at time $t=0, \ldots, T$, with $\omega^{1}, \ldots, \omega^{t} \in\{\mathrm{u}, \mathrm{d}\}$, will be identified with the event $\left\{\omega \in \Omega: \eta^{1}=\omega^{1}, \ldots, \eta^{t}=\right.$ $\left.\omega^{t}\right\}$. In particular, node $\omega_{0}$ will be identified with $\Omega$. The family of all nodes $\omega_{t}$ at time $t$ will be denoted by $\Omega_{t}$. We take a filtration $\{\emptyset, \Omega\}=\mathcal{F}_{0} \subset \mathcal{F}_{1} \subset$ $\ldots \subset \mathcal{F}_{T}=\mathcal{F}$, where $\mathcal{F}_{t}$ is the $\sigma$-field generated by the family $\Omega_{t}$ for each $t=0, \ldots, T$. We shall often identify $\mathcal{F}_{t}$-measurable random variables on $\Omega$ with functions defined on $\Omega_{t}$.

The market model will consist of a risk-free and a risky security, a bond (or cash) and a stock. Trading in stock is subject to proportional transaction costs. At any time $t=0, \ldots, T$ a share can be bought for the ask price $S_{t}^{a}$ or sold for the bid price $S_{t}^{b}$, where $S_{t}^{a} \geq S_{t}^{b}>0$. The price processes $S^{a}$ and $S^{b}$ are adapted to the filtration.

Without loss of generality we can assume the bond to be a risk-free security with zero interest rate, the bond price being 1 for all $t=0, \ldots, T$. Equivalently, all prices can be regarded as discounted prices.

\subsection{Self-Financing Strategies, Arbitrage, Risk-Neutral Measures}

The time $t=0, \ldots, T$ liquidation value $\vartheta_{t}$ of a portfolio $(\xi, \zeta)$ of cash and stock can be defined by

$$
\vartheta_{t}(\xi, \zeta)=\xi+\zeta^{+} S_{t}^{b}-\zeta^{-} S_{t}^{a} .
$$

Definition 2.1 By a self-financing strategy we shall understand a pair $(\alpha, \beta)$ of predictable processes $\alpha_{t}, \beta_{t}$ representing positions in cash and stock for $t=$ $0, \ldots, T$ such that $\beta_{0}=0$ and

$$
\vartheta_{t}\left(\alpha_{t}-\alpha_{t+1}, \beta_{t}-\beta_{t+1}\right) \geq 0
$$

for each $t=0, \ldots, T-1$. The set of such strategies will be denoted by $\Phi\left(S^{a}, S^{b}\right)$.

Observe that the self-financing condition (2.1) holds if and only if for each $u \in\{a, b\}$

$$
\alpha_{t}+\beta_{t} S_{t}^{u} \geq \alpha_{t+1}+\beta_{t+1} S_{t}^{u} .
$$

Definition 2.2 By an arbitrage opportunity we understand a strategy $(\alpha, \beta) \in$ $\Phi\left(S^{a}, S^{b}\right)$ such that $\alpha_{0} \leq 0, \vartheta_{T}\left(\alpha_{T}, \beta_{T}\right) \geq 0$ and $\mathbb{Q}\left\{\vartheta_{T}\left(\alpha_{T}, \beta_{T}\right)>0\right\}>0$. 
Definition 2.3 We say that a probability measure $\mathbb{P}$ equivalent to $\mathbb{Q}$ is a riskneutral measure if there is a martingale $S$ under $\mathbb{P}$ such that $S_{t}^{b} \leq S_{t} \leq S_{t}^{a}$ for each $t=0, \ldots, T$. By $\mathcal{S}$ we shall denote the set of such martingales $S$, and by $\mathcal{P}$ the set of the corresponding risk-neutral measures $\mathbb{P}$.

The following result, obtained by Jouini and Kallal [JK95, who used a slightly different notion of arbitrage, referred to as 'free lunch' in their work, is also valid under the above definition of an arbitrage opportunity in the present setting, as shown in Tokarz [Tok04]. See also Ortu Ort01].

Theorem 2.1 (Jouini and Kallal [JK95]) There is no arbitrage opportunity if and only if $\mathcal{P}$ is non-empty or, equivalently, $\mathcal{S}$ is non-empty.

\subsection{Small Proportional Transaction Costs}

For any $t=0, \ldots, T-1$ and any node $\omega_{t} \in \Omega_{t}$ the corresponding single-step subtree of stock prices can be depicted as

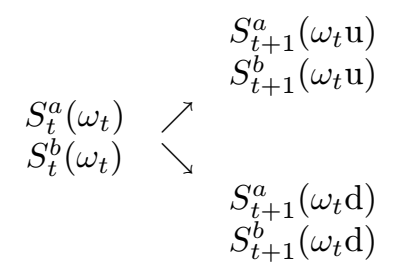

Throughout this paper we shall work under the following assumption, which means that the bid-ask spreads do not overlap in any single-step tree fragment as above.

Assumption (small proportional transaction costs) For each $t=$ $0, \ldots, T-1$ and each $\omega_{t} \in \Omega_{t}$

$$
S_{t+1}^{b}\left(\omega_{t} \mathrm{~d}\right) \leq S_{t+1}^{a}\left(\omega_{t} \mathrm{~d}\right)<S_{t}^{b}\left(\omega_{t}\right) \leq S_{t}^{a}\left(\omega_{t}\right)<S_{t+1}^{b}\left(\omega_{t} \mathrm{u}\right) \leq S_{t+1}^{a}\left(\omega_{t} \mathrm{u}\right) .
$$

It follows that $\mathcal{S}$ is non-empty. In particular, $S^{a}, S^{b} \in \mathcal{S}$. Consequently, the set of risk-neutral measures $\mathcal{P}$ is also non-empty, and no arbitrage opportunity exists under the small transaction costs assumption 2.2 .

\subsection{Notation}

Here we introduce some notation, which will be used throughout this paper. For any $u, v, w \in\{a, b\}$, any $t=0, \ldots, T-1$ and $\omega_{t} \in \Omega_{t}$, and any $\mathcal{F}_{t+1}$-measurable $\mathbb{R}^{2}$-valued random variables $\mathbf{G}=\left(G^{a}, G^{b}\right)$ and $\mathbf{H}=\left(H^{a}, H^{b}\right)$ we put

$$
\mathbb{E}_{t}^{u v w}\left(\mathbf{G} ; \mathbf{H} \mid \omega_{t}\right)=p_{t}^{u v w}\left(\omega_{t}\right) G^{v}\left(\omega_{t} \mathbf{u}\right)+\left(1-p_{t}^{u v w}\left(\omega_{t}\right)\right) H^{w}\left(\omega_{t} \mathrm{~d}\right),
$$

where

$$
p_{t}^{u v w}\left(\omega_{t}\right)=\frac{S_{t}^{u}\left(\omega_{t}\right)-S_{t+1}^{w}\left(\omega_{t} \mathrm{~d}\right)}{S_{t+1}^{v}\left(\omega_{t} \mathrm{u}\right)-S_{t+1}^{w}\left(\omega_{t} \mathrm{~d}\right)}
$$


We shall write $\mathbb{E}_{t}^{u v w}(\mathbf{G} ; \mathbf{H})$ to denote the $\mathcal{F}_{t}$-measurable random variable $\omega_{t} \mapsto$ $\mathbb{E}_{t}^{u v w}\left(\mathbf{G} ; \mathbf{H} \mid \omega_{t}\right)$.

This notation is slightly more complicated than necessary in the case of the writer of an American option, for which we shall always have $\mathbf{G}=\mathbf{H}$ in $\mathbb{E}_{t}^{u v w}\left(\mathbf{G} ; \mathbf{H} \mid \omega_{t}\right)$. However we need to allow $\mathbf{G} \neq \mathbf{H}$ when discussing the buyer's case for an American option.

It will sometimes prove convenient to write $\mathbf{S}_{t}=\left(S_{t}^{a}, S_{t}^{b}\right)$ for any $t=0, \ldots, T$. Observe that

$$
S_{t}^{u}=\mathbb{E}_{t}^{u v w}\left(\mathbf{S}_{t+1} ; \mathbf{S}_{t+1}\right)
$$

for each $u, v, w \in\{a, b\}$ and each $t=0, \ldots, T-1$.

If $G$ is an $\mathcal{F}_{t+1}$-measurable random variable for some $t=0, \ldots, T-1$, then we shall write $G(\mathrm{u})$ and $G(\mathrm{~d})$ to denote the $\mathcal{F}_{t}$-measurable random variables $\omega_{t} \mapsto G\left(\omega_{t} \mathrm{u}\right)$ and $\omega_{t} \mapsto G\left(\omega_{t} \mathrm{~d}\right)$, respectively.

\section{Ask and Bid Prices of American Options}

We shall consider an American option to be exercised by the delivery of a portfolio $\left(\xi_{\tau}, \zeta_{\tau}\right)$ of cash and stock at a stopping time $\tau$ chosen by the option buyer such that $0 \leq \tau \leq T$, where $(\xi, \zeta)$ is an $\mathbb{R}^{2}$-valued adapted process. By $\mathcal{T}$ we denote the family of stopping times $\tau$ such that $0 \leq \tau \leq T$. We shall refer to $(\xi, \zeta)$ as the payoff process and to $T$ as the expiry time.

Definition 3.1 The ask price (upper hedging price) and the bid price (lower hedging price) of an American option with payoff process $(\xi, \zeta)$ and expiry time $T$ are defined, respectively, by

$$
\begin{aligned}
& \pi^{a}(\xi, \zeta)=\min \left\{\alpha_{0} \mid(\alpha, \beta) \in \Phi\left(S^{a}, S^{b}\right), \forall \tau \in \mathcal{T}: \vartheta_{\tau}\left(\alpha_{\tau}-\xi_{\tau}, \beta_{\tau}-\zeta_{\tau}\right) \geq 0\right\} \\
& \pi^{b}(\xi, \zeta)=\max \left\{-\alpha_{0} \mid(\alpha, \beta) \in \Phi\left(S^{a}, S^{b}\right), \exists \tau \in \mathcal{T}: \vartheta_{\tau}\left(\alpha_{\tau}+\xi_{\tau}, \beta_{\tau}+\zeta_{\tau}\right) \geq 0\right\}
\end{aligned}
$$

The minimum and maximum are attained because the corresponding sets are closed and, respectively, bounded below and above in the discrete setting.

The ask and bid prices of options have important implications. First of all, they provide arbitrage bounds on the price at which options are traded under transaction costs: A writer who could sell an option $(\xi, \zeta)$ for more than the ask price $\pi^{a}(\xi, \zeta)$ would be able to achieve arbitrage, as would a buyer who paid less than the bid price $\pi^{b}(\xi, \zeta)$. Indeed $\pi^{a}(\xi, \zeta)$ and $\pi^{b}(\xi, \zeta)$ are the lowest and, respectively, the highest prices with this property. Moreover, $\pi^{a}(\xi, \zeta)$ and $\pi^{b}(\xi, \zeta)$ ensure liquidity in the options market. An option $(\xi, \zeta)$ can be purchased on demand for $\pi^{a}(\xi, \zeta)$ because any option writer who receives this amount will be able to hedge a short position in the option. Similarly, the option can be sold on demand for $\pi^{b}(\xi, \zeta)$ because any option buyer will be able to hedge a shorted amount $\pi^{b}(\xi, \zeta)$ against a long position in the option. As a result, $\pi^{a}(\xi, \zeta)$ and $\pi^{b}(\xi, \zeta)$ play a similar role in ensuring liquidity of options as the ask and bid prices $S^{a}$ and $S^{b}$ for stock. 
In what follows it will prove convenient to use processes $X^{a}, X^{b}$ such that for each $u \in\{a, b\}$ and each $t=0, \ldots, T$

$$
X_{t}^{u}=\xi_{t}+\zeta_{t} S_{t}^{u}
$$

Note that the condition $\vartheta_{\tau}\left(\alpha_{\tau}-\xi_{\tau}, \beta_{\tau}-\zeta_{\tau}\right) \geq 0$ in Definition 3.1 is equivalent to $\alpha_{\tau}+\beta_{\tau} S_{\tau}^{u} \geq X_{\tau}^{u}$ for each $u \in\{a, b\}$, and $\vartheta_{\tau}\left(\alpha_{\tau}+\xi_{\tau}, \beta_{\tau}+\zeta_{\tau}\right) \geq 0$ is equivalent to $\alpha_{\tau}+\beta_{\tau} S_{\tau}^{u} \geq-X_{\tau}^{u}$ for each $u \in\{a, b\}$.

\subsection{Ask Price Algorithm}

Algorithm 3.1 Given an American option with payoff process $(\xi, \zeta)$ and expiry time $T$ we construct an $\mathbb{R}^{2}$-valued process $\mathbf{Z}=\left(Z^{a}, Z^{b}\right)$ by backward induction:

- For each $u \in\{a, b\}$ we put

$$
Z_{T}^{u}=V_{T}^{u}=X_{T}^{u}
$$

- For each $t=1, \ldots, T$ and each $u \in\{a, b\}$ we put

$$
Z_{t-1}^{u}=\max \left\{X_{t-1}^{u}, V_{t-1}^{u}\right\}
$$

where

$$
V_{t-1}^{u}=\max _{v, w \in\{a, b\}} \mathbb{E}_{t-1}^{u v w}\left(\mathbf{Z}_{t} ; \mathbf{Z}_{t}\right)
$$

- The algorithm returns

$$
\max \left\{Z_{0}^{a}, Z_{0}^{b}\right\}
$$

shown in Theorem 3.2 to be the ask price $\pi^{a}(\xi, \zeta)$ of the option.

The processes $V^{a}, V^{b}$ correspond to the value of continuation in the standard Snell envelope construction, whereas $Z^{a}, Z^{b}$ correspond to the Snell envelope itself.

\subsection{Writer's Hedging Strategy}

Lemma 3.1 Let $t=1, \ldots, T$ and let $\gamma, \delta$ be $\mathcal{F}_{t-1}$-measurable random variables such that for each $u \in\{a, b\}$

$$
\gamma+\delta S_{t-1}^{u} \geq V_{t-1}^{u}
$$

Then there are $\mathcal{F}_{t-1}$-measurable random variables $\rho, \sigma$ such that for each $u \in$ $\{a, b\}$

$$
\begin{aligned}
\gamma+\delta S_{t-1}^{u} & \geq \rho+\sigma S_{t-1}^{u}, \\
\rho+\sigma S_{t}^{u} & \geq Z_{t}^{u} .
\end{aligned}
$$

Observe that the proof of this lemma in Appendix A.2 provides concrete formulae for $\rho$ and $\sigma$. With the aid of the lemma, we can construct by induction a strategy hedging the position of the writer of an American option $(\xi, \zeta)$ : 
- Put $\alpha_{0}=\max \left\{Z_{0}^{a}, Z_{0}^{b}\right\}$ and $\beta_{0}=0$. Then $\alpha_{0}, \beta_{0}$ are $\mathcal{F}_{0}$-measurable and for each $u \in\{a, b\}$

$$
\alpha_{0}+\beta_{0} S_{0}^{u} \geq Z_{0}^{u} \geq V_{0}^{u} .
$$

- For any $t=1, \ldots, T$, if $\mathcal{F}_{t-1}$-measurable random variables $\alpha_{t-1}, \beta_{t-1}$ such that for each $u \in\{a, b\}$

$$
\alpha_{t-1}+\beta_{t-1} S_{t-1}^{u} \geq V_{t-1}^{u}
$$

have already been constructed, then Lemma 3.1 provides $\mathcal{F}_{t-1}$-measurable random variables $\alpha_{t}, \beta_{t}$ such that for each $u \in\{a, b\}$

$$
\begin{aligned}
\alpha_{t-1}+\beta_{t-1} S_{t-1}^{u} & \geq \alpha_{t}+\beta_{t} S_{t-1}^{u}, \\
\alpha_{t}+\beta_{t} S_{t}^{u} & \geq Z_{t}^{u} \geq X_{t}^{u}=\xi_{t}+\zeta_{t} S_{t}^{u} .
\end{aligned}
$$

Since the random variables $\alpha_{t}, \beta_{t}$ are $\mathcal{F}_{t-1}$-measurable, they are also $\mathcal{F}_{t}$-measurable, and for $t<T$ we also have $\alpha_{t}+\beta_{t} S_{t}^{u} \geq Z_{t}^{u} \geq V_{t}^{u}$ for each $u \in\{a, b\}$, which makes it possible to iterate the last step.

As a result, we obtain a strategy $(\alpha, \beta) \in \Phi\left(S^{a}, S^{b}\right)$ such that $\vartheta_{\tau}\left(\alpha_{\tau}-\xi_{\tau}, \beta_{\tau}-\right.$ $\left.\zeta_{\tau}\right) \geq 0$ for each $\tau \in \mathcal{T}$, that is, a hedging strategy for the option writer. By Definition 3.1, it follows that

$$
\pi^{a}(\xi, \zeta) \leq \alpha_{0}=\max \left\{Z_{0}^{a}, Z_{0}^{b}\right\} .
$$

In Theorem 3.2 we shall prove that $(\alpha, \beta)$ is in fact an optimal hedging strategy for the writer, that is, $\pi^{a}(\xi, \zeta)=\alpha_{0}$.

\subsection{Representations for Ask Price}

The following theorem establishes three representations for the ask price of an American option: in terms of the writer's hedging strategy, the pricing algorithm, and the risk-neutral expectations of stopped payoffs. The latter representation resembles that proved by Cvitanić and Karatzas [CK96] in continuous time setting.

Theorem 3.2 Under the small transaction costs assumption (2.2) the ask price of an American option $(\xi, \zeta)$ can be represented as

$$
\begin{aligned}
\pi^{a}(\xi, \zeta) & =\alpha_{0} \\
& =\max \left\{Z_{0}^{a}, Z_{0}^{b}\right\} \\
& =\max _{\tau \in \mathcal{T}} \max _{S \in \mathcal{S}} \mathbb{E}\left(\xi_{\tau}+\zeta_{\tau} S_{\tau}\right),
\end{aligned}
$$

where $\alpha_{0}$ is the initial value of the writer's hedging strategy $(\alpha, \beta)$ constructed in Section $3.2, Z^{a}, Z^{b}$ are constructed in Algorithm 3.1, and $\mathbb{E}$ is the expectation under the probability measure $\mathbb{P}$ that turns $S$ into a martingale.

The proof of this theorem can be found in Appendix A.2. 


\subsection{Bid Price Algorithm}

Algorithm 3.2 Given an American option with payoff process $(\xi, \zeta)$ and expiry time $T$ we consider the $\mathbb{R}^{2}$-valued process $\mathbf{X}=\left(X^{a}, X^{b}\right)$ with $X^{a}, X^{b}$ given by $(3.1)$, and construct an $\mathbb{R}^{2}$-valued process $\mathbf{U}=\left(U^{a}, U^{b}\right)$ by backward induction as follows:

- For each $u \in\{a, b\}$ we put

$$
U_{T}^{u}=X_{T}^{u}
$$

- For each $t=1, \ldots, T$ and each $u \in\{a, b\}$ we put

$$
U_{t-1}^{u}=\max _{\mathbf{V}, \mathbf{W} \in\left\{\mathbf{X}_{t}, \mathbf{U}_{t}\right\}} \min _{v, w \in\{a, b\}} \mathbb{E}_{t-1}^{u v w}(\mathbf{V} ; \mathbf{W}) .
$$

- The algorithm returns

$$
\max \left\{\min \left\{X_{0}^{a}, X_{0}^{b}\right\}, \min \left\{U_{0}^{a}, U_{0}^{b}\right\}\right\},
$$

shown in Theorem 3.4 to be the bid price $\pi^{b}(\xi, \zeta)$ of the option.

The processes $U^{a}, U^{b}$ correspond to the value of continuation in the standard Snell envelope construction.

\subsection{Buyer's Hedging Strategy}

Let us define a stopping time $\check{\tau} \in \mathcal{T}$ by putting

$$
\check{\tau}=t \text { on } A_{t} \backslash\left(A_{0} \cup \cdots \cup A_{t-1}\right)
$$

for $t=0, \ldots, T$, where

$$
A_{0}=\left\{\min \left\{X_{0}^{a}, X_{0}^{b}\right\} \geq \min \left\{U_{0}^{a}, U_{0}^{b}\right\}\right\}
$$

and

$$
\begin{array}{r}
A_{t}=\left\{\eta^{t}=\mathrm{u}, \exists \mathbf{W} \in\left\{\mathbf{X}_{t}, \mathbf{U}_{t}\right\}: U_{t-1}^{u}=\min _{v, w \in\{a, b\}} \mathbb{E}_{t-1}^{u v w}\left(\mathbf{X}_{t} ; \mathbf{W}\right)\right\} \\
\cup\left\{\eta^{t}=\mathrm{d}, \exists \mathbf{W} \in\left\{\mathbf{X}_{t}, \mathbf{U}_{t}\right\}: U_{t-1}^{u}=\min _{v, w \in\{a, b\}} \mathbb{E}_{t-1}^{u v w}\left(\mathbf{W} ; \mathbf{X}_{t}\right)\right\}
\end{array}
$$

for $t=1, \ldots, T$. These sets satisfy $\Omega=A_{0} \cup \cdots \cup A_{T}$ and, by Proposition A.1. are in fact independent of $u \in\{a, b\}$.

Lemma 3.3 Let $t=1, \ldots, T$ and let $\gamma, \delta$ be $\mathcal{F}_{t-1}$-measurable random variables such that for each $u \in\{a, b\}$

$$
\gamma+\delta S_{t-1}^{u} \geq-U_{t-1}^{u} \text { on }\{\check{\tau}>t-1\} .
$$

Then there are $\mathcal{F}_{t-1}$-measurable random variables $\rho, \sigma$ such that for each $u \in$ $\{a, b\}$

$$
\begin{aligned}
& \gamma+\delta S_{t-1}^{u} \geq \rho+\sigma S_{t-1}^{u} \\
& \rho+\sigma S_{t}^{u} \geq-X_{t}^{u} \text { on }\{\check{\tau}=t\} \\
& \rho+\sigma S_{t}^{u} \geq-U_{t}^{u} \text { on }\{\check{\tau}>t\}
\end{aligned}
$$


The proof of this lemma in Appendix A.3 provides concrete formulae for $\rho$ and $\sigma$. With the aid of the lemma, we can construct a strategy hedging the position of the buyer of an American option $(\xi, \zeta)$ as follows:

- We put $\alpha_{0}=-\max \left\{\min \left\{X_{0}^{a}, X_{0}^{b}\right\}, \min \left\{U_{0}^{a}, U_{0}^{b}\right\}\right\}$ and $\beta_{0}=0$. Then for each $u \in\{a, b\}$

$$
\alpha_{0}+\beta_{0} S_{0}^{u} \geq-U_{0}^{u} \text { on }\{\check{\tau}>0\} .
$$

- For any $t=1, \ldots, T$, if $\mathcal{F}_{t-1}$-measurable random variables $\alpha_{t-1}, \beta_{t-1}$ such that for each $u \in\{a, b\}$

$$
\alpha_{t-1}+\beta_{t-1} S_{t-1}^{u} \geq-U_{t-1}^{u} \text { on }\{\check{\tau}>t-1\}
$$

have already been constructed, then Lemma 3.3 provides $\mathcal{F}_{t-1}$-measurable random variables $\alpha_{t}, \beta_{t}$ such that for each $u \in\{a, b\}$

$$
\begin{aligned}
\alpha_{t-1}+\beta_{t-1} S_{t-1}^{u} & \geq \alpha_{t}+\beta_{t} S_{t-1}^{u}, \\
\alpha_{t}+\beta_{t} S_{t}^{u} & \geq-X_{t}^{u} \text { on }\{\check{\tau}=t\}, \\
\alpha_{t}+\beta_{t} S_{t}^{u} & \geq-U_{t}^{u} \text { on }\{\check{\tau}>t\} .
\end{aligned}
$$

Since the random variables $\alpha_{t}, \beta_{t}$ are $\mathcal{F}_{t-1}$-measurable, they are also $\mathcal{F}_{t}$-measurable. As a result, the last step can be iterated.

We have thus constructed a strategy $(\alpha, \beta) \in \Phi\left(S^{a}, S^{b}\right)$ such that $\vartheta_{\check{\tau}}\left(\alpha_{\check{\tau}}+\xi_{\check{\tau}}, \beta_{\check{\tau}}+\right.$ $\left.\zeta_{\check{\tau}}\right) \geq 0$, that is, a hedging strategy for the option buyer. By Definition 3.1, it follows that

$$
\pi^{b}(\xi, \zeta) \geq-\alpha_{0}=\max \left\{\min \left\{X_{0}^{a}, X_{0}^{b}\right\}, \min \left\{U_{0}^{a}, U_{0}^{b}\right\}\right\} .
$$

In Theorem 3.4 we shall prove that $(\alpha, \beta)$ is in fact an optimal hedging strategy for the buyer, that is, $\pi^{b}(\xi, \zeta)=-\alpha_{0}$.

\subsection{Representations for Bid Price}

As in the case of the ask price, representations of the bid price of an American option can be established in terms of the corresponding hedging strategy, the pricing algorithm, and the risk-neutral expectations of stopped payoffs. The proof of the following theorem can be found in Appendix A.3.

Theorem 3.4 Under the small transaction costs assumption (2.2) the bid price of an American option $(\xi, \zeta)$ can be expressed as

$$
\begin{aligned}
\pi^{b}(\xi, \zeta) & =-\alpha_{0} \\
& =\max \left\{\min \left\{X_{0}^{a}, X_{0}^{b}\right\}, \min \left\{U_{0}^{a}, U_{0}^{b}\right\}\right\} \\
& =\max _{\tau \in \mathcal{T}} \min _{S \in \mathcal{S}} \mathbb{E}\left(\xi_{\tau}+\zeta_{\tau} S_{\tau}\right),
\end{aligned}
$$

where $\alpha_{0}$ is the initial value of the buyer's hedging strategy $(\alpha, \beta)$ constructed in Section 3.5, $U^{a}, U^{b}$ are constructed in Algorithm 3.2, $X^{a}, X^{b}$ are given by (3.1), and where $\mathbb{E}$ is the expectation under the probability measure $\mathbb{P}$ that turns $S$ into a martingale. 
The representation of the bid price of the option in terms of risk-neutral expectations of stopped payoffs resembles that in Föllmer and Schied [FS02] (Theorem 6.33), where it applies to an incomplete friction-free market. However, in contrast to the latter, the maximum and minimum cannot, in general, be interchanged, i.e. no minimax theorem holds under transaction costs.

\section{Numerical Examples}

We shall apply Algorithms 3.1 and 3.2 to compute the ask and bid prices of American call options in the same setting as used in Boyle and Vorst BV92 for European calls under transaction costs. Computing American call prices is by no means a vacuous exercise, as it turns out that they are not necessarily equal to the European counterparts in the presence of transaction costs.

Following the approach in Boyle and Vorst BV92], we consider the CoxRoss-Rubinstein binary tree model with parameters $u, d, r$ such that

$$
1+u=e^{\sigma \sqrt{h}}, \quad 1+d=e^{-\sigma \sqrt{h}}, \quad 1+r=\left(1+r_{e}\right)^{h},
$$

where $h=\frac{1}{N}, N$ is the number of time steps, $\sigma$ is the stock volatility per annum, and $r_{e}$ is the annually compounded interest rate (the effective rate). The stock price process $S$ is assumed to satisfy $S_{n+1}=\left(1+\varepsilon_{n}\right) S_{n}$ for $n=$ $0,1, \ldots, N-1$ with $S_{0}>0$, where $\varepsilon_{n}$ is a sequence of independent identically distributed random variables that take only two values, $1+u$ and $1+d$, both with positive probability.

Introducing the transaction cost rate $k$ as in Boyle and Vorst BV92, we express the bid and ask stock prices at any $n=1, \ldots, N$ as $S_{n}^{b}=S_{n}(1-k)$ and $S_{n}^{a}=S_{n}(1+k)$. Following [BV92], we also assume that there are no transaction costs at the initial time 0 , that is, $S_{0}^{b}=S_{0}^{a}=S_{0}$.

The small transaction costs assumption 2.2 is satisfied whenever $\frac{1+k}{1-k}<$ $e^{\sigma \sqrt{h}}\left(1+r_{e}\right)^{-h}$. For small values of $k$ and $h$, if the left- and right-hand sides are expanded to within terms of order $k$ and $\sqrt{h}$, this becomes $\frac{2 k}{\sigma \sqrt{h}}<1$. A similar restriction applies to the work by Leland [Lel85] extended by Hoggard, Whalley and Wilmott [HWW94, as discussed in detail by Avellaneda and Parás [AP94. (In our notation $2 k$ is what Avellaneda and Parás denote by $k$, the round-trip transaction cost rate.)

To compare our results with those obtained by Boyle and Vorst BV92 we consider an American call option with strike price $K$ and time to expiry $N h=1$ year, exercised by the delivery of a portfolio $\left(-K 1_{\{S \geq K\}}, 1_{\{S \geq K\}}\right)$ of cash and stock, with the following input parameters: the initial stock price $S_{0}=100$, stock volatility $\sigma=20 \%$ p.a., and effective interest rate $r_{e}=10 \%$.

The bid and ask prices of American and also European call options on the same stock for various levels of small proportional transaction costs and strike prices are presented in the table below. All numerical calculations for American options are based on Algorithms 3.1 and 3.2, whereas those for European options on the algorithm in Boyle and Vorst BV92. The numbers in brackets represent the prices of European calls that differ from their American counterparts. 


\begin{tabular}{|c|c|c|c|c|c|c|c|c|}
\hline \multirow{3}{*}{$\begin{array}{c}\text { Strike } \\
\text { Price } \\
(K)\end{array}$} & \multicolumn{8}{|c|}{ Number of trading times $(N)$} \\
\hline & 6 & 6 & 13 & 13 & 52 & 52 & 250 & 250 \\
\hline & bid & ask & bid & ask & bid & ask & bid & ask \\
\hline & \multicolumn{8}{|c|}{$k=0 \%$} \\
\hline 80 & 27.703 & 27.703 & 27.701 & 27.701 & 27.665 & 27.665 & 27.675 & 27.675 \\
\hline 90 & 19.821 & 19.821 & 19.740 & 19.740 & 19.667 & 19.667 & 19.674 & 19.674 \\
\hline 100 & 12.665 & 12.665 & 13.093 & 13.093 & 12.953 & 12.953 & 12.984 & 12.984 \\
\hline 110 & 8.129 & 8.129 & 8.026 & 8.026 & 7.972 & 7.792 & 7.965 & 7.965 \\
\hline \multirow[t]{2}{*}{120} & 4.216 & 4.216 & 4.427 & 4.427 & 4.548 & 4.548 & 4.551 & 4.551 \\
\hline & \multicolumn{8}{|c|}{$k=0.125 \%$} \\
\hline 80 & 27.671 & 27.735 & 27.656 & 27.747 & 27.582 & 27.753 & 27.502 & 27.876 \\
\hline 90 & 19.749 & 19.894 & 19.638 & 19.842 & 19.469 & 19.865 & 19.246 & 20.103 \\
\hline \multirow[t]{2}{*}{100} & 12.555 & 12.770 & 12.935 & 13.248 & 12.644 & 13.256 & 12.288 & 13.630 \\
\hline & $(12.538)$ & & & & $(12.638)$ & & $(12.286)$ & \\
\hline 110 & 8.003 & 8.254 & 7.843 & 8.205 & 7.604 & 8.324 & 7.136 & 8.715 \\
\hline \multirow[t]{2}{*}{120} & 4.102 & 4.329 & 4.256 & 4.595 & 4.202 & 4.882 & 3.773 & 5.269 \\
\hline & \multicolumn{8}{|c|}{$k=0.5 \%$} \\
\hline 80 & 27.582 & 27.837 & 27.534 & 27.894 & 27.386 & 28.047 & 27.273 & 28.574 \\
\hline 90 & 10531 & 20113 & 10333 & 140 14 & $\begin{aligned}(27.383) \\
18.80\end{aligned}$ & 20453 & 18222 & 2134 \\
\hline 90 & 19.031 & 20.110 & 19.035 & 20.149 & 10.089 & 20.400 & $\begin{array}{l}10.224 \\
(18.221)\end{array}$ & ט \\
\hline 100 & 12.251 & 13.107 & 12.445 & 13.699 & 11.644 & 14.111 & 9.751 & 15.339 \\
\hline & $(12.177)$ & & & & (11.606) & & $(9.684)$ & \\
\hline 110 & 7.614 & 8.618 & 7.269 & 8.721 & 6.374 & 9.300 & 3.647 & 10.649 \\
\hline 120 & 3.754 & 4.663 & 3.726 & 5.084 & 3.077 & 5.820 & 0.879 & 7.161 \\
\hline
\end{tabular}

\subsection{American Versus European Bid and Ask Option Prices}

We can see in the table above that in the presence of proportional transaction costs the bid prices of American and European calls are not necessarily the same.

To illustrate this with an example that can readily be analysed by hand, consider a model with all input parameters the same as above, but with the number of time steps $N=2$, transaction cost rate $k=0.5 \%$ and strike price $K=100$. Here, the bid prices of the American and European call options are 11.838 and 11.729 , respectively. The optimal stopping time $\check{\tau}$ for the buyer of the American call turns out to be at nodes uu and ud after 2 time steps, and at node $\mathrm{d}$ after just 1 time step, that is, prior to option expiry. This realises the maximum over stopping times in Theorem 3.4 . On the other hand, the optimal stopping time $\hat{\tau}$ for the writer of the American call, which realises the maximum in Theorem 3.2, coincides with the expiry time.

It is interesting to observe that the two optimal stopping times, $\check{\tau}$ for the buyer and $\hat{\tau}$ for the writer of the American call do not have to be the same. Although a rational buyer can be expected to exercise the option at time $\check{\tau}$, to ensure that the writer's position is fully hedged it is necessary to account for the possibility that the buyer will behave in a suboptimal way and exercise at expiry rather than at $\check{\tau}$. It might prove beneficial for the parties to agree in advance 
that the option will in fact be exercised at time $\check{\tau}$, as the writer would then be able to charge a lower premium, a situation bearing some similarities to a Nash equilibrium.

As regards the ask prices of American and European calls, the following proposition holds in the general setting of Section 2 in the presence of a possibly non-zero risk-free return $r \geq 0$ over each time step, in which case the small transaction costs assumption 2.2 applies to discounted stock prices. The proposition will be proved in Appendix A.4.

Proposition 4.1 Suppose that $r \geq 0$, the discounted ask and bid stock prices satisfy (2.2), and $S$ is an adapted process such that $S_{t}^{b} \leq S_{t} \leq S_{t}^{a}$ for each $t=$ $0,1, \ldots, T$. Consider American and European calls with the same strike price $K$ and expiry $T$, the American option with payoff process $\left(-K 1_{\left\{S_{t} \geq K\right\}}, 1_{\left\{S_{t} \geq K\right\}}\right)$ for $t=0,1, \ldots, T$, and the European one with payoff $\left(-K 1_{\left\{S_{T} \geq K\right\}}, 1_{\left\{S_{T} \geq K\right\}}\right)$. Then the ask prices of these two options are the same.

\section{Conclusions and Outlook}

We have established a procedure for computing the ask price $\pi^{a}(\xi, \zeta)$ of an American contingent claim $(\xi, \zeta)$ under small proportional transaction costs in the binary tree setting, resembling the standard construction of the Snell envelope. A suitable algorithm has also been developed for computing the bid price $\pi^{b}(\xi, \zeta)$. Moreover, we have constructed optimal strategies to hedge long and short positions in American options, and established representations of the ask and bid option prices in terms of the initial values of these strategies, and also in terms of risk-neutral expectations of stopped payoffs. In addition, optimal stopping times $\hat{\tau}$ for the writer and $\check{\tau}$ for the buyer of the option have been constructed.

A distinctive feature of the algorithms developed under small transaction costs is that two quantities need to be tracked at each tree node, rather than a single one as in the standard iterative construction of the Snell envelope in the friction-free case.

In the numerical examples the algorithms have been applied to compute the ask and bid prices of American options in a realistic setting (option expiry $T=1$ year, daily rehedging of portfolios with $N=250$, and round-trip transaction costs $2 k=1 \%$ typical of large capitalisation stocks) satisfying the small transaction costs condition. The numerical results are compared to the earlier work by Boyle and Vorst BV92 for European options under proportional transaction costs. The small transaction costs assumption $(2.2)$ is shown to lead to a very similar restriction on the time step versus transaction cost rate as in Leland type approaches.

We have observed that the bid prices of American and European calls are not necessarily the same, and proved that the ask prices are equal to one another. Moreover, the optimal stopping times may be different for traders hedging long and short positions in American options under transaction costs. 
A natural question arises as to what will happen if the small costs assumption 2.2 is relaxed, so that only the no-arbitrage condition prevails. Results in Tokarz [Tok04, which apply to European options only, suggest that the algorithms for American options might need to be modified by keeping track of more than two quantities at each tree node whenever the bid-ask spreads for the stock price at adjacent nodes overlap. The work of Chalasani and Jha [CJ01. indicates that the constructions might also need to accommodate randomised stopping times in place of ordinary ones.

\section{Appendix: Proofs of Main Results}

\section{A.1 Auxiliary Results}

First of all, we shall state and outline the proofs of two technical propositions and a lemma, which are needed to prove the main results of this paper. The full proofs of the two propositions, which are elementary but somewhat tedious, can be found in Tok04.

Proposition A.1 Under assumption 2.2 , for any $t=1, \ldots, T$, any $\mathbb{R}^{2}$-valued $\mathcal{F}_{t}$-measurable random variables $\mathbf{A}=\left(A^{a}, A^{b}\right)$ and $\mathbf{B}=\left(B^{a}, B^{b}\right)$ and any $c, d \in$ $\{a, b\}$ the following conditions are equivalent:
(a) $\min _{v, w \in\{a, b\}} \mathbb{E}_{t-1}^{a v w}(\mathbf{A} ; \mathbf{B})=\mathbb{E}_{t-1}^{a c d}(\mathbf{A} ; \mathbf{B})$,
(b) $\min _{v, w \in\{a, b\}} \mathbb{E}_{t-1}^{b v w}(\mathbf{A} ; \mathbf{B})=\mathbb{E}_{t-1}^{b c d}(\mathbf{A} ; \mathbf{B})$.

The conditions remain equivalent to one another if the two minima are replaced by maxima.

Proof Outline The main steps of the proof are:

- For any $c, d, e, f, u \in\{a, b\}$ such that $c \neq e$ and $d \neq f$ show that the inequalities

$$
\begin{aligned}
& \mathbb{E}_{t-1}^{u c d}(\mathbf{A} ; \mathbf{B}) \leq \mathbb{E}_{t-1}^{u e d}(\mathbf{A} ; \mathbf{B}), \\
& \mathbb{E}_{t-1}^{u c d}(\mathbf{A} ; \mathbf{B}) \leq \mathbb{E}_{t-1}^{u c f}(\mathbf{A} ; \mathbf{B})
\end{aligned}
$$

imply

$$
\mathbb{E}_{t-1}^{u c d}(\mathbf{A} ; \mathbf{B}) \leq \mathbb{E}_{t-1}^{u e f}(\mathbf{A} ; \mathbf{B}),
$$

and deduce that A.1, A.2 are equivalent to

$$
\min _{v, w \in\{a, b\}} \mathbb{E}_{t-1}^{u v w}(\mathbf{A} ; \mathbf{B})=\mathbb{E}_{t-1}^{u c d}(\mathbf{A} ; \mathbf{B}) .
$$

- Verify that for any $c, d, e, f \in\{a, b\}$ the inequalities (A.1), A.2 with $u=a$ are equivalent to A.1, A.2 with $u=b$. 
The equivalence of (a) and (b) follows directly from these two steps.

Proposition A.2 Under assumption 2.2 , for any $t=1, \ldots, T$, any $\mathbb{R}^{2}$-valued $\mathcal{F}_{t}$-measurable random variables $\mathbf{A}=\left(A^{a}, A^{b}\right)$ and $\mathbf{B}=\left(B^{a}, B^{b}\right)$ and any $\mathbf{C}, \mathbf{D} \in\{\mathbf{A}, \mathbf{B}\}$ the following conditions are equivalent:

(a) $\max _{\mathbf{V}, \mathbf{W} \in\{\mathbf{A}, \mathbf{B}\}} \min _{v, w \in\{a, b\}} \mathbb{E}_{t-1}^{a v w}(\mathbf{V} ; \mathbf{W})=\min _{v, w \in\{a, b\}} \mathbb{E}_{t-1}^{a v w}(\mathbf{C} ; \mathbf{D})$,

(b) $\max _{\mathbf{V}, \mathbf{W} \in\{\mathbf{A}, \mathbf{B}\}} \min _{v, w \in\{a, b\}} \mathbb{E}_{t-1}^{b v w}(\mathbf{V} ; \mathbf{W})=\min _{v, w \in\{a, b\}} \mathbb{E}_{t-1}^{b v w}(\mathbf{C} ; \mathbf{D})$.

Proof Outline The main steps of the proof are:

- For any $\mathbf{C}, \mathbf{D}, \mathbf{E}, \mathbf{F} \in\{\mathbf{A}, \mathbf{B}\}$ such that $\mathbf{C} \neq \mathbf{E}$ and $\mathbf{D} \neq \mathbf{F}$ and for any $u \in\{a, b\}$ show that the inequalities

$$
\begin{aligned}
& \min _{v, w \in\{a, b\}} \mathbb{E}_{t-1}^{u v w}(\mathbf{C} ; \mathbf{D}) \geq \min _{v, w \in\{a, b\}} \mathbb{E}_{t-1}^{u v w}(\mathbf{E} ; \mathbf{D}), \\
& \min _{v, w \in\{a, b\}} \mathbb{E}_{t-1}^{u v w}(\mathbf{C} ; \mathbf{D}) \geq \min _{v, w \in\{a, b\}} \mathbb{E}_{t-1}^{u v w}(\mathbf{C} ; \mathbf{F})
\end{aligned}
$$

imply

$$
\min _{v, w \in\{a, b\}} \mathbb{E}_{t-1}^{u v w}(\mathbf{C} ; \mathbf{D}) \geq \min _{v, w \in\{a, b\}} \mathbb{E}_{t-1}^{u v w}(\mathbf{E} ; \mathbf{F}),
$$

and deduce that A.3, A.4 are equivalent to

$$
\max _{\mathbf{V}, \mathbf{W} \in\{\mathbf{A}, \mathbf{B}\}} \min _{v, w \in\{a, b\}} \mathbb{E}_{t-1}^{u v w}(\mathbf{V} ; \mathbf{W})=\min _{v, w \in\{a, b\}} \mathbb{E}_{t-1}^{u v w}(\mathbf{C} ; \mathbf{D}) .
$$

- Verify that for any $\mathbf{C}, \mathbf{D}, \mathbf{E}, \mathbf{F} \in\{\mathbf{A}, \mathbf{B}\}$ the inequalities $(\mathrm{A} .3)$, A.4 with $u=a$ are equivalent to A.3, A.4 with $u=b$.

The equivalence of (a) and (b) follows directly from these two steps.

Lemma A.3 If $(\alpha, \beta) \in \Phi\left(S^{a}, S^{b}\right)$ and $S \in \mathcal{S}$ is a martingale under $\mathbb{P} \in \mathcal{P}$, then $\alpha+\beta S$ is a supermartingale under $\mathbb{P}$.

Proof of Lemma A.3 Since $S_{t}^{b} \leq S_{t} \leq S_{t}^{a}$, the self-financing condition 2.1 implies that

$$
\alpha_{t}-\alpha_{t+1} \geq\left(\beta_{t}-\beta_{t+1}\right)^{-} S_{t}^{a}-\left(\beta_{t}-\beta_{t+1}\right)^{+} S_{t}^{b} \geq-\left(\beta_{t}-\beta_{t+1}\right) S_{t} .
$$

As a result, for each $t=0, \ldots, T-1$

$$
\mathbb{E}\left(\alpha_{t+1}+\beta_{t+1} S_{t+1} \mid \mathcal{F}_{t}\right)=\alpha_{t+1}+\beta_{t+1} S_{t} \leq \alpha_{t}+\beta_{t} S_{t},
$$

where $\mathbb{E}$ denotes the expectation under $\mathbb{P}$. 


\section{A.2 Results for Ask Price}

Proof of Lemma 3.1 By Proposition A.1 there are $g, h \in\{a, b\}$ such that $V_{t-1}^{u}=\mathbb{E}_{t-1}^{u g h}\left(\mathbf{Z}_{t} ; \mathbf{Z}_{t}\right)$ for each $u \in\{a, b\}$. We put

$$
\sigma=\frac{Z_{t}^{g}(\mathrm{u})-Z_{t}^{h}(\mathrm{~d})}{S_{t}^{g}(\mathrm{u})-S_{t}^{h}(\mathrm{~d})}, \quad \rho=Z_{t}^{g}(\mathrm{u})-\sigma S_{t}^{g}(\mathrm{u})=Z_{t}^{h}(\mathrm{~d})-\sigma S_{t}^{h}(\mathrm{~d}) .
$$

Then, for each $u \in\{a, b\}$

$$
\rho+\sigma S_{t-1}^{u}=\rho+\sigma \mathbb{E}_{t-1}^{u g h}\left(\mathbf{S}_{t} ; \mathbf{S}_{t}\right)=\mathbb{E}_{t-1}^{u g h}\left(\mathbf{Z}_{t} ; \mathbf{Z}_{t}\right)=V_{t-1}^{u} \leq \gamma+\delta S_{t-1}^{u},
$$

where we have used (a) in the last inequality. Next, observe that for each $r \in\{a, b\}$

$$
\mathbb{E}_{t-1}^{u g h}\left(\mathbf{Z}_{t} ; \mathbf{Z}_{t}\right) \geq \mathbb{E}_{t-1}^{u r h}\left(\mathbf{Z}_{t} ; \mathbf{Z}_{t}\right), \quad \mathbb{E}_{t-1}^{u g h}\left(\mathbf{Z}_{t} ; \mathbf{Z}_{t}\right) \geq \mathbb{E}_{t-1}^{u g r}\left(\mathbf{Z}_{t} ; \mathbf{Z}_{t}\right),
$$

which can be transformed, respectively, into

$$
\rho+\sigma S_{t}^{r}(\mathrm{u}) \geq Z_{t}^{r}(\mathrm{u}), \quad \rho+\sigma S_{t}^{r}(\mathrm{~d}) \geq Z_{t}^{r}(\mathrm{~d}) .
$$

This gives $\rho+\sigma S_{t}^{r} \geq Z_{t}^{r}$ for each $r \in\{a, b\}$, as required.

Proof of Theorem 3.2 We begin by constructing processes $\hat{S}, \hat{V}, \hat{Z}$ such that:

- For some $u \in\{a, b\}$

$$
\begin{aligned}
& \hat{S}_{0}=S_{0}^{u}, \\
& \hat{V}_{0}=V_{0}^{u}, \\
& \hat{Z}_{0}=Z_{0}^{u},
\end{aligned}
$$

and

$$
Z_{0}^{u}=\max \left\{Z_{0}^{a}, Z_{0}^{b}\right\}
$$

- For each $t=0, \ldots, T-1$ and each $\omega_{t} \in \Omega_{t}$ there are $v, w \in\{a, b\}$ such that

$$
\begin{array}{ll}
\hat{S}_{t+1}\left(\omega_{t} \mathrm{u}\right)=S_{t+1}^{v}\left(\omega_{t} \mathrm{u}\right), & \hat{S}_{t+1}\left(\omega_{t} \mathrm{~d}\right)=S_{t+1}^{w}\left(\omega_{t} \mathrm{~d}\right), \\
\hat{V}_{t+1}\left(\omega_{t} \mathrm{u}\right)=V_{t+1}^{v}\left(\omega_{t} \mathrm{u}\right), & \hat{V}_{t+1}\left(\omega_{t} \mathrm{~d}\right)=V_{t+1}^{w}\left(\omega_{t} \mathrm{~d}\right), \\
\hat{Z}_{t+1}\left(\omega_{t} \mathrm{u}\right)=Z_{t+1}^{v}\left(\omega_{t} \mathrm{u}\right), & \hat{Z}_{t+1}\left(\omega_{t} \mathrm{~d}\right)=Z_{t+1}^{w}\left(\omega_{t} \mathrm{~d}\right),
\end{array}
$$

and

$$
V_{t}^{u}\left(\omega_{t}\right)=\mathbb{E}_{t}^{u v w}\left(\mathbf{Z}_{t+1} ; \mathbf{Z}_{t+1} \mid \omega_{t}\right)
$$

for each $u \in\{a, b\}$. Such $v, w$ exist by Proposition A.1.

The processes $\hat{S}, \hat{V}, \hat{Z}$ may not be unique. The lack of uniqueness may arise whenever there is more than one pair $v, w \in\{a, b\}$ satisfying (A.6), or there is more than one $u \in\{a, b\}$ such that A.5 holds. In such cases we can choose any $\hat{S}, \hat{V}, \hat{Z}$ satisfying the conditions above.

Because of the small transaction costs assumption $2.2, \hat{S} \in \mathcal{S}$. Let $\hat{\mathbb{P}} \in \mathcal{P}$ be the probability measure turning $\hat{S}$ into a martingale, and let $\hat{\mathbb{E}}$ denote the expectation under $\hat{\mathbb{P}}$. 
We claim that $\hat{Z}$ is the Snell envelope of the process $\xi+\zeta \hat{S}$ under $\hat{\mathbb{P}}$. Indeed, by the construction of $\hat{S}, \hat{V}, \hat{Z}$, for any $t=0, \ldots, T-1$ and any $\omega_{t} \in \Omega_{t}$ there are $u, v, w \in\{a, b\}$ such that

$$
\begin{array}{lll}
\hat{S}_{t}\left(\omega_{t}\right)=S_{t}^{u}\left(\omega_{t}\right), & \hat{S}_{t+1}\left(\omega_{t} \mathrm{u}\right)=S_{t+1}^{v}\left(\omega_{t} \mathrm{u}\right), & \hat{S}_{t+1}\left(\omega_{t} \mathrm{~d}\right)=S_{t+1}^{w}\left(\omega_{t} \mathrm{~d}\right), \\
\hat{V}_{t}\left(\omega_{t}\right)=V_{t}^{u}\left(\omega_{t}\right), & \hat{V}_{t+1}\left(\omega_{t} \mathrm{u}\right)=V_{t+1}^{v}\left(\omega_{t} \mathrm{u}\right), & \hat{V}_{t+1}\left(\omega_{t} \mathrm{~d}\right)=V_{t+1}^{w}\left(\omega_{t} \mathrm{~d}\right), \\
\hat{Z}_{t}\left(\omega_{t}\right)=Z_{t}^{u}\left(\omega_{t}\right), & \hat{Z}_{t+1}\left(\omega_{t} \mathrm{u}\right)=Z_{t+1}^{v}\left(\omega_{t} \mathrm{u}\right), & \hat{Z}_{t+1}\left(\omega_{t} \mathrm{~d}\right)=Z_{t+1}^{w}\left(\omega_{t} \mathrm{~d}\right),
\end{array}
$$

and (A.6) holds. Thus

$$
\hat{V}_{t}\left(\omega_{t}\right)=V_{t}^{u}\left(\omega_{t}\right)=\mathbb{E}_{t}^{u v w}\left(\mathbf{Z}_{t+1} ; \mathbf{Z}_{t+1} \mid \omega_{t}\right)=\hat{\mathbb{E}}\left(\hat{Z}_{t+1} \mid \omega_{t}\right) .
$$

Because $Z_{T}^{u}=\xi_{T}+\zeta_{T} S_{T}^{u}$ for each $u \in\{a, b\}$, we have $\hat{Z}_{T}=\xi_{T}+\zeta_{T} \hat{S}_{T}$. Since $Z_{t}^{u}=\max \left\{\xi_{t}+\zeta_{t} S_{t}^{u}, V_{t}^{u}\right\}$ for each $u \in\{a, b\}$, it follows that

$$
\hat{Z}_{t}=\max \left\{\xi_{t}+\zeta_{t} \hat{S}_{t}, \hat{V}_{t}\right\}=\max \left\{\xi_{t}+\zeta_{t} \hat{S}_{t}, \hat{\mathbb{E}}\left(\hat{Z}_{t+1} \mid \mathcal{F}_{t}\right)\right\}
$$

for each $t=0, \ldots, T-1$, proving the claim.

Next, we define a stopping time $\hat{\tau} \in \mathcal{T}$ by

$$
\hat{\tau}=\min \left\{t \mid \hat{Z}_{t}=\xi_{t}+\zeta_{t} \hat{S}_{t}\right\} .
$$

Because $\hat{Z}$ is the Snell envelope of $\xi+\zeta \hat{S}$ under $\hat{\mathbb{P}}$ it follows that

$$
\hat{\mathbb{E}}\left(\xi_{\hat{\tau}}+\zeta_{\hat{\tau}} \hat{S}_{\hat{\tau}}\right)=\hat{Z}_{0}=\max \left\{Z_{0}^{a}, Z_{0}^{b}\right\}
$$

This proves that

$$
\max \left\{Z_{0}^{a}, Z_{0}^{b}\right\} \leq \max _{\tau \in \mathcal{T}} \max _{S \in \mathcal{S}} \mathbb{E}\left(\xi_{\tau}+\zeta_{\tau} S_{\tau}\right)
$$

Finally, take a strategy $(\alpha, \beta) \in \Phi\left(S^{a}, S^{b}\right)$ such that $\alpha_{0}=\pi^{a}(\xi, \zeta)$ and $\vartheta_{\tau}\left(\alpha_{\tau}-\xi_{\tau}, \beta_{\tau}-\zeta_{\tau}\right) \geq 0$ for each $\tau \in \mathcal{T}$. Then $\xi_{\tau}+\zeta_{\tau} S_{\tau}^{u} \leq \alpha_{\tau}+\beta_{\tau} S_{\tau}^{u}$ for each $u \in\{a, b\}$ and each $\tau \in \mathcal{T}$. Also, take any $S \in \mathcal{S}$. Because $S_{\tau}^{b} \leq S_{\tau} \leq S_{\tau}^{a}$ it follows that $\xi_{\tau}+\zeta_{\tau} S_{\tau} \leq \alpha_{\tau}+\beta_{\tau} S_{\tau}$. By Lemma A.3 the process $\alpha+\beta S$ is a supermartingale under the probability measure $\mathbb{P} \in \mathcal{P}$ that turns $S$ into a martingale. As a result,

$$
\mathbb{E}\left(\xi_{\tau}+\zeta_{\tau} S_{\tau}\right) \leq \mathbb{E}\left(\alpha_{\tau}+\beta_{\tau} S_{\tau}\right) \leq \alpha_{0}+\beta_{0} S_{0}=\alpha_{0}=\pi^{a}(\xi, \zeta),
$$

where $\mathbb{E}$ is the expectation under $\mathbb{P}$. Since $S \in \mathcal{S}$ and $\tau \in \mathcal{T}$ are arbitrary,

$$
\max _{\tau \in \mathcal{T}} \max _{S \in \mathcal{S}} \mathbb{E}\left(\xi_{\tau}+\zeta_{\tau} S_{\tau}\right) \leq \pi^{a}(\xi, \zeta) .
$$

Inequalities (A.7) and (A.8) together with (3.3) complete the proof. 


\section{A.3 Results for Bid Price}

Proof of Lemma 3.3 Take $\mathbf{G}, \mathbf{H} \in\left\{\mathbf{X}_{t}, \mathbf{U}_{t}\right\}$ and $g, h \in\{a, b\}$ such that for each $u \in\{a, b\}$

$$
U_{t-1}^{u}=\min _{v, w \in\{a, b\}} \mathbb{E}_{t-1}^{u v w}(\mathbf{G} ; \mathbf{H})=\mathbb{E}_{t-1}^{u g h}(\mathbf{G} ; \mathbf{H}) .
$$

Such $\mathbf{G}, \mathbf{H}$ and $g, h$ exist by Propositions A.1 and A.2. We put

$$
\sigma=0, \quad \rho=\gamma+\delta^{+} S_{t-1}^{b}-\delta^{-} S_{t-1}^{a}
$$

on $\{\check{\tau}<t\}$, and

$$
\sigma=-\frac{G^{g}(\mathrm{u})-H^{h}(\mathrm{~d})}{S_{t}^{g}(\mathrm{u})-S_{t}^{h}(\mathrm{~d})}, \quad \rho=-G^{g}(\mathrm{u})-\sigma S_{t}^{g}(\mathrm{u})=-H^{h}(\mathrm{~d})-\sigma S_{t}^{h}(\mathrm{~d})
$$

on $\{\check{\tau} \geq t\}$. Then, for each $u \in\{a, b\}$

$$
\rho+\sigma S_{t-1}^{u}=\gamma+\delta^{+} S_{t-1}^{b}-\delta^{-} S_{t-1}^{a} \leq \gamma+\delta S_{t-1}^{u}
$$

on $\{\check{\tau}<t\}$, and

$$
\rho+\sigma S_{t-1}^{u}=\rho+\sigma \mathbb{E}_{t-1}^{u g h}\left(\mathbf{S}_{t} ; \mathbf{S}_{t}\right)=-\mathbb{E}_{t-1}^{u g h}(\mathbf{G} ; \mathbf{H})=-U_{t-1}^{u} \leq \gamma+\delta S_{t-1}^{u}
$$

on $\{\check{\tau} \geq t\}$. Next, observe that for each $r \in\{a, b\}$

$$
\mathbb{E}_{t-1}^{u g h}(\mathbf{G} ; \mathbf{H}) \leq \mathbb{E}_{t-1}^{u r h}(\mathbf{G} ; \mathbf{H}), \quad \mathbb{E}_{t-1}^{u g h}(\mathbf{G} ; \mathbf{H}) \leq \mathbb{E}_{t-1}^{u g r}(\mathbf{G} ; \mathbf{H}),
$$

which can be transformed, respectively, into

$$
\rho+\sigma S_{t}^{r}(\mathrm{u}) \geq-G^{r}(\mathrm{u}), \quad \rho+\sigma S_{t}^{r}(\mathrm{~d}) \geq-H^{r}(\mathrm{~d})
$$

on $\{\check{\tau} \geq t\}$. By the construction of $\check{\tau}$ we know that $\mathbf{G}=\mathbf{H}=\mathbf{U}_{t}$ on $\{\check{\tau}>t\}$, and we can select $\mathbf{G}=\mathbf{X}_{t}$ on $\left\{\check{\tau}=t, \eta^{t}=\mathrm{u}\right\}$ and $\mathbf{H}=\mathbf{X}_{t}$ on $\left\{\check{\tau}=t, \eta^{t}=\mathrm{d}\right\}$. It follows that $\rho+\sigma S_{t}^{r} \geq-X_{t}^{r}$ on $\{\check{\tau}=t\}$ and $\rho+\sigma S_{t}^{r} \geq-U_{t}^{r}$ on $\{\check{\tau}>t\}$ for each $r \in\{a, b\}$, as required.

Proof of Theorem 3.4 We take any $\tau \in \mathcal{T}$ and a number $C$ such that for each $u \in\{a, b\}$ and each $t=0, \ldots, T$

$$
C>X_{t}^{u} \text {. }
$$

Consider the processes $Z^{a}, Z^{b}$ constructed as in Algorithm 3.1 for the American option with exercise time $T$ and payoff process $(\kappa, \lambda)$ defined by

$$
\left(\kappa_{t}, \lambda_{t}\right)= \begin{cases}\left(-\xi_{\tau},-\zeta_{\tau}\right) & \text { on }\{\tau=t\} \\ (-C, 0) & \text { on }\{\tau \neq t\}\end{cases}
$$

for each $t=0, \ldots, T$. We claim that for each $u \in\{a, b\}$ and each $t=0, \ldots, T$

$$
Z_{t}^{u} \geq-U_{t}^{u} \text { on }\{\tau>0\} \text {. }
$$

This can be proved by backward induction on $t$. Since $\{\tau>T\}$ is empty, the claim is trivially satisfied for $t=T$. Now suppose that the claim is valid for some $t=1, \ldots, T$. For each node $\omega_{t-1} \in \Omega_{t-1}$ such that $\tau>t-1$ at $\omega_{t-1}$ there are up to four possibilities: 
1. If $\tau>t$ at $\omega_{t-1} \mathrm{u}$ and at $\omega_{t-1} \mathrm{~d}$, then $Z_{t}^{v} \geq-U_{t}^{v}$ at $\omega_{t-1} \mathrm{u}$ and $Z_{t}^{w} \geq-U_{t}^{w}$ at $\omega_{t-1} \mathrm{~d}$ for each $v, w \in\{a, b\}$, so at $\omega_{t-1}$ for each $u \in\{a, b\}$

$$
Z_{t-1}^{u} \geq \max _{v, w \in\{a, b\}} \mathbb{E}_{t-1}^{u v w}\left(\mathbf{Z}_{t} ; \mathbf{Z}_{t}\right) \geq-\min _{v, w \in\{a, b\}} \mathbb{E}_{t-1}^{u v w}\left(\mathbf{U}_{t} ; \mathbf{U}_{t}\right) \geq-U_{t-1}^{u} ;
$$

2. If $\tau=t$ at $\omega_{t-1} \mathrm{u}$ and $\tau>t$ at $\omega_{t-1} \mathrm{~d}$, then $Z_{t}^{v}=-X_{t}^{v}$ at $\omega_{t-1} \mathrm{u}$ and $Z_{t}^{w} \geq-U_{t}^{w}$ at $\omega_{t-1} \mathrm{~d}$ for each $v, w \in\{a, b\}$, so at $\omega_{t-1}$ for each $u \in\{a, b\}$

$$
Z_{t-1}^{u} \geq \max _{v, w \in\{a, b\}} \mathbb{E}_{t-1}^{u v w}\left(\mathbf{Z}_{t} ; \mathbf{Z}_{t}\right) \geq-\min _{v, w \in\{a, b\}} \mathbb{E}_{t-1}^{u v w}\left(\mathbf{X}_{t} ; \mathbf{U}_{t}\right) \geq-U_{t-1}^{u} ;
$$

3. If $\tau>t$ at $\omega_{t-1} \mathrm{u}$ and $\tau=t$ at $\omega_{t-1} \mathrm{~d}$, then $Z_{t}^{v} \geq-U_{t}^{v}$ at $\omega_{t-1} \mathrm{u}$ and $Z_{t}^{w}=-X_{t}^{w}$ at $\omega_{t-1} \mathrm{~d}$ for each $v, w \in\{a, b\}$, so at $\omega_{t-1}$ for each $u \in\{a, b\}$

$$
Z_{t-1}^{u} \geq \max _{v, w \in\{a, b\}} \mathbb{E}_{t-1}^{u v w}\left(\mathbf{Z}_{t} ; \mathbf{Z}_{t}\right) \geq-\min _{v, w \in\{a, b\}} \mathbb{E}_{t-1}^{u v w}\left(\mathbf{U}_{t} ; \mathbf{X}_{t}\right) \geq-U_{t-1}^{u}
$$

4. Finally, if $\tau=t$ at $\omega_{t-1} \mathrm{u}$ and at $\omega_{t-1} \mathrm{~d}$, then $Z_{t}^{v}=-X_{t}^{v}$ at $\omega_{t-1} \mathrm{u}$ and $Z_{t}^{w}=-X_{t}^{w}$ at $\omega_{t-1} \mathrm{~d}$ for each $v, w \in\{a, b\}$, so at $\omega_{t-1}$ for each $u \in\{a, b\}$

$$
Z_{t-1}^{u} \geq \max _{v, w \in\{a, b\}} \mathbb{E}_{t-1}^{u v w}\left(\mathbf{Z}_{t} ; \mathbf{Z}_{t}\right)=-\min _{v, w \in\{a, b\}} \mathbb{E}_{t-1}^{u v w}\left(\mathbf{X}_{t} ; \mathbf{X}_{t}\right) \geq-U_{t-1}^{u} .
$$

This verifies the claim. It follows that $Z_{0}^{u} \geq-U_{0}^{u}$ on $\{\tau>0\}$ for each $u \in\{a, b\}$. We also have $Z_{0}^{u}=-X_{0}^{u}$ on $\{\tau=0\}$ for each $u \in\{a, b\}$. As a result,

$$
-\max \left\{Z_{0}^{a}, Z_{0}^{b}\right\} \leq \max \left\{\min \left\{X_{0}^{a}, X_{0}^{b}\right\}, \min \left\{U_{0}^{a}, U_{0}^{b}\right\}\right\} .
$$

Observe that on $\{t \neq \tau\}$

$$
\kappa_{t}+\lambda_{t} S_{t}=-C<-\max \left\{X_{\tau}^{a}, X_{\tau}^{b}\right\} \leq-\xi_{\tau}-\zeta_{\tau} S_{\tau}=\kappa_{\tau}+\lambda_{\tau} S_{\tau}
$$

for any $S \in \mathcal{S}$. Consequently, $\mathbb{E}\left(\kappa_{\sigma}+\lambda_{\sigma} S_{\sigma}\right) \leq \mathbb{E}\left(\kappa_{\tau}+\lambda_{\tau} S_{\tau}\right)$ for each $\sigma \in \mathcal{T}$ and $S \in \mathcal{S}$, where $\mathbb{E}$ is the expectation under the probability measure $\mathbb{P} \in \mathcal{P}$ that turns $S$ into a martingale. Thus, by Theorem 3.2

$$
\max \left\{Z_{0}^{a}, Z_{0}^{b}\right\}=\max _{\sigma \in \mathcal{T}} \max _{S \in \mathcal{S}} \mathbb{E}\left(\kappa_{\sigma}+\lambda_{\sigma} S_{\sigma}\right)=\max _{S \in \mathcal{S}} \mathbb{E}\left(\kappa_{\tau}+\lambda_{\tau} S_{\tau}\right)=-\min _{S \in \mathcal{S}} \mathbb{E}\left(\xi_{\tau}+\zeta_{\tau} S_{\tau}\right) .
$$

It follows that for each $\tau \in \mathcal{T}$

$$
\min _{S \in \mathcal{S}} \mathbb{E}\left(\xi_{\tau}+\zeta_{\tau} S_{\tau}\right) \leq \max \left\{\min \left\{X_{0}^{a}, X_{0}^{b}\right\}, \min \left\{U_{0}^{a}, U_{0}^{b}\right\}\right\},
$$

so that

$$
\max _{\tau \in \mathcal{T}} \min _{S \in \mathcal{S}} \mathbb{E}\left(\xi_{\tau}+\zeta_{\tau} S_{\tau}\right) \leq \max \left\{\min \left\{X_{0}^{a}, X_{0}^{b}\right\}, \min \left\{U_{0}^{a}, U_{0}^{b}\right\}\right\} .
$$

Next take any $S \in \mathcal{S}$ and a strategy $(\alpha, \beta) \in \Phi\left(S^{a}, S^{b}\right)$ such that $\alpha_{0}=$ $-\pi^{b}(\xi, \zeta)$ and there is a $\tau \in \mathcal{T}$ such that $\vartheta_{\tau}\left(\alpha_{\tau}+\xi_{\tau}, \beta_{\tau}+\zeta_{\tau}\right) \geq 0$, and therefore $-\xi_{\tau}-\zeta_{\tau} S_{\tau}^{u} \leq \alpha_{\tau}+\beta_{\tau} S_{\tau}^{u}$ for each $u \in\{a, b\}$. Because $S_{\tau}^{b} \leq S_{\tau} \leq S_{\tau}^{a}$ it follows that $-\xi_{\tau}-\zeta_{\tau} S_{\tau} \leq \alpha_{\tau}+\beta_{\tau} S_{\tau}$. By Lemma A.3 the process $\alpha+\beta S$ is 
a supermartingale under the probability measure $\mathbb{P} \in \mathcal{P}$ that turns $S$ into a martingale. As a result,

$$
\mathbb{E}\left(-\xi_{\tau}-\zeta_{\tau} S_{\tau}\right) \leq \mathbb{E}\left(\alpha_{\tau}+\beta_{\tau} S_{\tau}\right) \leq \alpha_{0}+\beta_{0} S_{0}=\alpha_{0}=-\pi^{b}(\xi, \zeta),
$$

where $\mathbb{E}$ is the expectation under $\mathbb{P}$. Since $S \in \mathcal{S}$ is arbitrary,

$$
\pi^{b}(\xi, \zeta) \leq \min _{S \in \mathcal{S}} \mathbb{E}\left(\xi_{\tau}+\zeta_{\tau} S_{\tau}\right)
$$

which implies that

$$
\pi^{b}(\xi, \zeta) \leq \max _{\tau \in \mathcal{T}} \min _{S \in \mathcal{S}} \mathbb{E}\left(\xi_{\tau}+\zeta_{\tau} S_{\tau}\right)
$$

Inequalities $(\mathrm{A} .9)$ and $\mathrm{A} .10 \mathrm{~T}$ together with $(3.4)$ complete the proof.

\section{A.4 Equality of Ask Prices of American and European Calls}

For an American call with expiry $T$ and payoff process $\left(\xi_{t}, \zeta_{t}\right)=$ $\left(-K 1_{\left\{S_{t} \geq K\right\}}, 1_{\left\{S_{t} \geq K\right\}}\right)$, where $t=0,1, \ldots, T$, we denote by $\mathbf{X}=\left(X^{a}, X^{b}\right)$ the process defined by (3.1), and by $\mathbf{Z}^{A}=\left(Z^{A a}, Z^{A b}\right)$ the process constructed in Algorithm 3.1 modified by the inclusion of the factor $(1+r)^{-1}$ multiplying the right-hand side of $(3.2)$ and the factor $(1+r)$ multiplying $S_{t}^{u}\left(\omega_{t}\right)$ in (2.3) to account for the presence of a possibly non-zero risk-free return $r \geq 0$. Similarly, by $\mathbf{Z}^{E}=\left(Z^{E a}, Z^{E b}\right)$ we denote the analogous process for a European call with payoff $\left(\xi_{T}, \zeta_{T}\right)=\left(-K 1_{\left\{S_{T} \geq K\right\}}, 1_{\left\{S_{T} \geq K\right\}}\right)$ and expiry $T$, which can for this purpose be regarded as an American option with payoff process

$$
\left(\kappa_{t}, \lambda_{t}\right)= \begin{cases}(0,0) & \text { for } t=0,1, \ldots, T-1, \\ \left(-K 1_{\left\{S_{T} \geq K\right\}}, 1_{\left\{S_{T} \geq K\right\}}\right) & \text { for } t=T .\end{cases}
$$

Proof of Proposition 4.1 First, we claim that

$$
Z_{t}^{E u} \geq\left(S_{t}^{u}-K\right)^{+}
$$

for each $u \in\{a, b\}$ and each $t=0,1, \ldots, T-1$, which we shall prove by backward induction on $t$. Observe that for each $u \in\{a, b\}$

$$
\begin{aligned}
Z_{T-1}^{E u} & =(1+r)^{-1} \max _{v, w \in\{a, b\}} E_{T-1}^{u v w}\left(\mathbf{X}_{T} ; \mathbf{X}_{T}\right) \geq(1+r)^{-1} E_{T-1}^{u b b}\left(\mathbf{X}_{T} ; \mathbf{X}_{T}\right) \\
& \geq S_{T-1}^{u}-(1+r)^{-1} K \geq S_{T-1}^{u}-K,
\end{aligned}
$$

since $X_{T}^{b}=\left(S_{T}^{b}-K\right) 1_{\left\{S_{T} \geq K\right\}} \geq S_{T}^{b}-K$. Moreover, $X_{T}^{a}=\left(S_{T}^{a}-K\right) 1_{\left\{S_{T} \geq K\right\}} \geq 0$, so

$$
Z_{T-1}^{E u}=(1+r)^{-1} \max _{v, w \in\{a, b\}} E_{T-1}^{u v w}\left(\mathbf{X}_{T} ; \mathbf{X}_{T}\right) \geq(1+r)^{-1} E_{T-1}^{u a a}\left(\mathbf{X}_{T} ; \mathbf{X}_{T}\right) \geq 0 .
$$


The claim has been proved for $t=T-1$. Now take some $t=1, \ldots, T-1$ and suppose that $Z_{t}^{E u} \geq\left(S_{t}^{u}-K\right)^{+}$for each $u \in\{a, b\}$. Then, $Z_{t}^{E u} \geq S_{t}^{u}-K$ for each $u \in\{a, b\}$ and therefore

$$
Z_{t-1}^{E u}=(1+r)^{-1} \max _{v, w \in\{a, b\}} E_{t-1}^{u v w}\left(\mathbf{Z}_{t}^{E} ; \mathbf{Z}_{t}^{E}\right) \geq S_{t}^{u}-(1+r)^{-1} K \geq S_{t}^{u}-K .
$$

Because $Z_{t}^{E u} \geq 0$ for each $u \in\{a, b\}$, it follows that $Z_{t-1}^{E u}=(1+$ $r)^{-1} \max _{v, w} E_{t-1}^{u v w}\left(Z_{t}^{v} ; Z_{t}^{w}\right) \geq 0$ for each $u \in\{a, b\}$. As a result, $Z_{t-1}^{E u} \geq$ $\left(S_{t}^{u}-K\right)^{+}$for each $u \in\{a, b\}$, completing the proof of claim (A.11).

To prove the equality of the American and European call prices it suffices to verify that for each $u \in\{a, b\}$ and each $t=0,1, \ldots, T$

$$
Z_{t}^{A u}=Z_{t}^{E u}
$$

This can be verified by backward induction on $t$. For $t=T$ we have $Z_{T}^{A u}=$ $X_{T}^{u}=Z_{T}^{E u}$. Suppose that A.12 holds for some $t=1, \ldots, T$. Then

$$
\begin{aligned}
Z_{t-1}^{A u} & =\max \left\{X_{t-1}^{u},(1+r)^{-1} \max _{v, w \in\{a, b\}} E_{t-1}^{u v w}\left(\mathbf{Z}_{t}^{A} ; \mathbf{Z}_{t}^{A}\right)\right\} \\
& =\max \left\{X_{t-1}^{u},(1+r)^{-1} \max _{v, w \in\{a, b\}} E_{t-1}^{u v w}\left(\mathbf{Z}_{t}^{E} ; \mathbf{Z}_{t}^{E}\right)\right\} \\
& =\max \left\{X_{t-1}^{u}, Z_{t-1}^{E u}\right\}=Z_{t-1}^{E u},
\end{aligned}
$$

where the last equality holds because, by A.11,

$$
Z_{t-1}^{E u} \geq\left(S_{t-1}^{u}-K\right)^{+} \geq\left(S_{t-1}^{u}-K\right) 1_{\left\{S_{t-1} \geq K\right\}}=X_{t-1}^{u},
$$

completing the proof.

\section{References}

[AP94] M. Avellaneda and A. Parás, Dynamic hedging portfolios for derivative securities in the presence of large transaction costs, Appl. Math. Finance 1 (1994), 165-193.

[BLPS92] B. Bensaid, J. Lesne, H. Pagès, and J. Scheinkman, Derivative asset pricing with transaction costs, Math. Finance 2 (1992), 63-86.

[BV92] P. Boyle and T. Vorst, Option replication in discrete time with transaction costs, J. Finance 47 (1992), 271-293.

[CJ01] P. Chalasani and S. Jha, Randomized stopping times and American option pricing with transaction costs, Math. Finance 1 (2001), 33-77.

[CK96] J. Cvitanić and I. Karatzas, Hedging and portfolio optimization under transaction costs: a martinangle approach, Math. Finance 6 (1996), 133-166. 
[CP04] G.M. Constantinides and S. Perrakis, Stochastic dominance bounds on American option prices in markets with frictions, Working paper, University of Chicago, 2004.

[CPT99] J. Cvitanić, H. Pham, and N Touzi, A closed-form solution to the problem of super-replication under transaction costs, Finance Stoch. 3 (1999), 35-54.

[CZ01] G.M. Constantinides and T. Zariphopoulou, Bounds on derivative prices in an intertemporal setting with proportional transaction costs and multiple securities, Math. Finance 11 (2001), 331-346.

[DR91] J.C. Dermody and R.T. Rockafellar, Cash stream valuation in the face of transaction costs and taxes, Math. Finance 1 (1991), 31-54.

[DZ95] M.H.A. Davis and T. Zariphopoulou, American options and transaction fees, Mathematical Finance (M.H.A. Davis et al., eds.), IMA Volumes in Mathematics and Its Applications, vol. 65, Springer, New York, 1995, pp. 47-61.

[ENU93] C. Edirsinghe, V. Naik, and R. Uppal, Optimal replication of options with transactions costs and trading restrictions, J. Fin. Quant. Anal. 28 (1993), 117-138.

[FS02] H. Föllmer and A. Schied, Stochastic Finance. An Introduction in Discrete Time, Walter de Gruyter, Berlin, 2002.

[HWW94] T. Hoggard, A.E. Whalley, and P. Wilmott, Hedging option portfolios in the presence of transaction costs, Adv. Futures Options Res. 7 (1994), 21-35.

[JK95] E. Jouini and H. Kallal, Martingales and arbitrage in securities markets with transaction costs, J. Econom. Theory 66 (1995), 178-197.

[JLR03] P. Jakubenas, S. Levental, and M. Ryznar, The super-replication problem via probabilistic methods, Ann. Appl. Probab. 13 (2003), $742-773$.

[Jou00] E. Jouini, Price functionals with bid-ask spreads: An axiomatic approach, J. Math. Econom. 34 (2000), 547-558.

[Koc99] M. Kociński, Optimality of the replicating strategy for American options, Appl. Math. (Warsaw) 26 (1999), 93-105.

[Koc01]__ Pricing of the American option in discrete time with proportional transaction costs, Math. Methods Oper. Res. 53 (2001), $67-88$.

[Koc04]_ Hedging of the European option in discrete time under proportional transaction costs, Math. Methods Oper. Res. 59 (2004), $315-328$. 
[KPT96] P.-F. Koehl, H. Pham, and N. Touzi, Option pricing under transaction costs: A martingale approach, Institut National de la Statistique et des Etudes Economiques, Working paper 9647, 1996.

[KPT99] - Hedging in discrete time under transaction costs and continuous-time limit, J. Appl. Probab. 36 (1999), 163-178.

[KPT01] - On super-replication in discrete time under transaction costs, Theory Probab. Appl. 47 (2001), 667-673.

[Kus95] S. Kusuoka, Limit theorem on option replication cost with transaction costs, Ann. Appl. Probab. 5 (1995), 198-221.

[Lel85] H.E. Leland, Option pricing and replication with transaction costs, J. Finance 40 (1985), 1283-1301.

[LS97] S. Levental and A.V. Skorohod, On the possibility of hedging options in the presence of transaction costs, Ann. Appl. Probab. 7 (1997), 410-443.

[Mer90] R.C. Merton, Continuous Time Finance, Blackwell, Oxford, 1990.

[MP05] A.V. Melnikov and Y.G. Petrachenko, On option pricing in binomial market with transaction costs, Finance Stoch. 9 (2005), 141-149.

[MV97] F. Mercurio and T.C.F. Vorst, Options pricing and hedging in discrete time with transaction costs, Mathematics of Derivative Securities (M.A.H. Dempster and S.R. Pliska, eds.), Cambridge University Press, Cambridge, UK, 1997, pp. 190-215.

[Nai95] V. Naik, Finite state securities, market models and arbitrage, Handbooks in Operations Research and Management Science, Vol. 9, Finance (R Jarrow et al., eds.), Elsevier, Amsterdam, 1995, pp. 31-64.

[Ort01] F. Ortu, Arbitrage, linear programming and martingales in securities markets with bid-ask spreads, Decis. Econom. Finance 24 (2001), no. 2, 79-105.

[Pal01a] K. Palmer, A note on the Boyle-Vorst discrete-time option pricing model with transaction costs, Math. Finance 11 (2001), 357-363.

[Pal01b] _ Replicating and super-replicating portfolios in the BoyleVorst discrete-time option pricing model with transaction costs, Working paper, University of Melbourne, 2001.

[PL97] S. Perrakis and J. Lefoll, Derivative asset pricing with transaction costs: an extension, Comput. Econom. 10 (1997), 359-376.

[PL00] _ Option pricing and replication with transaction costs and dividends, J. Econom. Dynam. Control 24 (2000), 1527-1561. 
[PL04] - The American put under transaction costs, J. Econom. Dynam. Control 28 (2004), 915-935.

[Rut98] M. Rutkowski, Optimality of replication in the CRR model with transaction costs, Appl. Math. (Warsaw) 25 (1998), 29-53.

[SK95] H. Shirakawa and H. Konno, Pricing of options under the proportional transaction costs, Preprint, Tokyo Institute of Technology, 1995.

[SSC95] H.M. Soner, S.E. Shreve, and J. Cvitanić, There is no nontrivial hedging portfolio for option pricing with transaction costs, Ann. Appl. Probab. 5 (1995), 327-355.

[Ste97] L. Stettner, Option pricing in the CRR model with proportional transaction costs: A cone transformation approach, Appl. Math. (Warsaw) 24 (1997), 475-514.

[Ste00] _ Option pricing in discrete-time incomplete market models, Math. Finance 10 (2000), 305-321.

[Tok04] K. Tokarz, European and American option pricing under proportional transaction costs, Ph.D. thesis, University of Hull, 2004.

[Tou99] N. Touzi, Super-replication under proportional transaction costs: From discrete to continuous-time models, Math. Meth. Oper. Res. 50 (1999), 297-320. 